\title{
On a relation between the Fitting length of a soluble group and the number of conjugacy classes of its maximal nilpotent subgroups
}

\section{H. Lausch and A. Makan}

In a finite soluble group $G$, the Fitting (or nilpotency) length $h(G)$ can be considered as a measure for how strongly $G$ deviates from being nilpotent. As another measure for this, the number $v(G)$ of conjugacy classes of the maximal nilpotent subgroups of $G$ may be taken. It is shown that there exists an integer-valued function $f$ on the set of positive integers such that $h(G) \leqq f(v(G)$ ) for all finite (soluble) groups of odd order. Moreover, if all prime divisors of the order of $G$ are greater than $\nu(G)(v(G)-1) / 2$, then $h(G) \leqq 3$. The bound $f(v(G))$ is just of qualitative nature and by far not best possible. For $v(G)=2, h(G)=3$, some statements are made about the structure of $G$.

1. In various papers by Gross [3], Hoffman [5], and Thompson [9], bounds were given for the nilpotency length of a finite soluble group in terms of the group exponent (in the case of certain pq-groups) the order of a fixed-point-free p-automorphism, or of the number of primes (not necessarily different) dividing the order of a soluble, $\pi$ '-automorphism group of a $\pi$-group. The main result of this paper is

THEOREM. Let $h(G)$ be the Fitting length of a finite group $G$ of odd order, $v(G)$ the number of different conjugacy classes of the maximal

Received 27 January 1969 
nilpotent subgroups of $G$, then there exists a function $f$, defined on the set of natural numbers and taking natural nombers as values, such that $h(G) \leqq f(\nu(G))$.

Rose [8] has, however, shown that there is no lower bound for $h(G)$ in terms of $\nu(G)$. The statement of the theorem is merely of qualitative nature, for $\nu(G)=2,3$ sharp bounds are given, and for $\nu(G)=2$, $h(G)=3$ some statements are made about the structure of $G$. All the groups are assumed to be finite and soluble in this paper.

2. In order to prove the theorem, we start with some lemmas.

LEMMA 1. If $N \triangleleft G$, then $v(G / N) \leqq V(G)$. Every maximal nilpotent subgroup of $G / N$ can be written as $V N / N$ where $V$ is maximal nilpotent in $G$. If $\nu(G / N)=v(G)$, and $V$ is maximal nilpotent in $G$, then $V N / N$ is maximal nilpotent in $G / N$.

Proof. Let $W / N$ be maximal nilpotent in $G / N$. Then there exists a nilpotent subgroup $W_{1}$ in $G$ such that $W=W_{1} N$. Let $V$ be a maximal nilpotent subgroup of $G$ such that $W_{1} \subset V$. Then $W / N=W_{1} N / N \subset V N / N$ whence $W=V N$ by maximality of $W / N$. The other statements of the lemma follow.

LEMMA 2. Let $N \triangleleft G, N$ nilpotent, $V$ maximal nilpotent in $G$, $V \phi N, W \subset V, \quad(|W|,|N|)=1$, and $W \neq \bar{V}$ for every nilpotent subgroup $\bar{V}$ of $G$ containing $N$. Then either $C_{N}(W)=V \cap N$ or there exists $a$ maximal nilpotent subgroup $T$ of $G, T \neq N$ such that $W \subset T$, $V \cap N<T \cap N=C_{N}(W)$. If, in particular, $V \cap N$ is maximal among $\{T \cap N \mid T$ maximal nilpotent in $G, T \neq N\}$, then $C_{N}(W)=V \cap N$.

Proof. Since $(|W|,|N|)=1$ we have $V \cap N \subset C_{N}(W)$, and $C_{N}(W) \times W$ is a nilpotent subgroup of $G$. Let $T$ be a maximal nilpotent subgroup of $G$ such that $T \supset C_{N}(W) \times W$. Then $T \not N$ as $W \subset T$, by assumption. Also $(|W|,|N|)=1$ implies $T \cap N \subset C_{N}(W)$ and so $T \cap N=C_{N}(W)$. The second statement of Lemma 2 is then obvious.

LEMMA 3. Let $F$ be the Fitting subgroup of $G,|G|$ odd, and suppose $F$ is an elementaxy-abelian p-group. Let $V$ be a maximal nilpotent subgroup of $G, V \downarrow F$, and $Z$ be the largest integer for 
which there exists a chain of subgroups $V \cap F<v_{l-1} \cap F<v_{\eta-2} \cap F<\ldots<$ $V_{1} \cap F<F$ where $V_{i}$ is a maximal nilpotent subgroup of $G$ for $i=1,2, \ldots, l-1$. Then, for every prime $q \neq p$, every abelian $q$-subgroup of $V$ can be generated by at most $l$ elements.

Proof. By induction on $Z$. For $Z=1$, let $U$ be an abelian q-subgroup of $V, z \in U$. By Lemma 2, $V \cap F=C_{F}(z)$ implying $U$ is cyclic, otherwise $F=\prod_{z \in U \backslash\{1\}} C_{F}(z)=V \cap F$ (see [2], Th. 6.2.4). Suppose Lema 3 is true for all integers less than $Z$, and let $U$ be an abelian q-subgroup of $V$. Let $\underline{\underline{K}}=\left\{C_{F}(T) \mid\{1\}<T \subset U\right\} . \underline{\underline{K}}$ is ordered by inclusion. Either $\underline{\underline{K}}$ consists just of one element, then, again by [2], Th. 6.2.4, $U$ is cyclic, or we can choose a second minimal element $C_{F}(R)=X \cap F$ in $\underline{\underline{K}}$, where $\{1\}<R<U$ and $X$ is maximal nilpotent in $G$, by Lemma 2. $C_{U}(X \cap F) \times(X \cap F)$ is nilpotent, hence $C_{U}(X \cap F) \subset Y, X \cap F \subset Y \cap F$ for some maximal nilpotent subgroup $Y$ of $G$. Also $V \cap F \subset C_{F}(U)<C_{F}(R)=X \cap F$ whence $V \cap F<Y \cap F$ so that, by induction, $C_{U}(X \cap F)$ can be generated by at most $l-1$ elements. It remains to show that $U / C_{U}(X \cap F)$ is cyclic. If $u \in U$, then $(X \cap F)^{u}=C_{F}\left(R^{u}\right)=C_{F}(R)=X \cap F$, and so $U \subset N_{G}(X \cap F)$. We claim $U / C_{U}(X \cap F)$ acts faithfully on $X \cap F / C_{F}(U)$. Let $u \in U$, and $[u, X \cap F] \subset C_{F}(U)$. By Maschke's theorem, $X \cap F=C_{F}(U) \times L$ where $L$ is a $U$-module. Hence $[u, L] \subset C_{F}(U) \cap L=1$, and so $u \in C_{U}(L)=$ $C_{U}(X \cap F)$. We claim that $U / C_{U}(X \cap F)$ acts in a fixed-point-free manner on $X \cap F / C_{F}(U)$. For, let $u \in U, x \in X \cap F,[u, x] \in C_{F}(U)$. We may write $x=y z, y \in C_{F}(U), z \in L$. Then $[u, z] \in C_{E}(U) \cap L=1$ and $z \in C_{F}(u)$. If $C_{F}(u) \ngtr X \cap F$, then $X \cap F>C_{F}(u) \cap(X \cap F)=$ $C_{F}(u) \cap C_{F}(R)=C_{F}(\langle u, R\rangle)=C_{F}(U)$, by definition of $X \cap F$. Hence $z \in C_{F}(U)$ and so $x \in C_{F}(U)$. If $C_{F}(u) \supset X \cap F$, then $u \in C_{U}(X \cap F)$. Therefore $U / C_{U}(X \cap F)$ is cyclic.

LEMMA 4 (Thompson). If $G$ is a p-group, $p>2$ and every abelian 
normal subgroup of $G$ can be generated by $k$ elements, then every subgroup of $G$ can be generated by $\frac{k(k+1)}{2}$ elements.

Proof. See [6], III. Satz 12.3.

LEMMA 5 (Huppert). Let $G$ be a p-soluble group, $V$ a vector space of dimension $n$ over $\mathrm{GF}(p)$, and let $G$ be faithfully and irreducibly represented on $V,(n,|G|)=1$. Then $G$ is cyclic and $|G| \mid p^{n}-1$.

Proof. See [7].

LEMMA 6. Let $G$ possess a Fitting subgroup $F$ such that $F$ is the unique minimal normal subgroup of $G$ and suppose $F$ is a p-group. Then there exists a normal subgroup $S$ of $G$ such that $h(G / S)=h(G)-1$, for $h(G)>1$. Moreover, the Fitting subgroup of $G / S$ is the unique minimal normal subgroup of $G / S$ and is a $p^{\prime}$-group.

Proof. Let $\underline{\underline{N}}$ be the class of nilpotent groups, $\underline{\underline{N}}^{0}=\{\{1\}\}$ and $\underline{\underline{N}}^{k}=\underline{\underline{N}}^{k-1} \underline{\underline{N}}$, for $k=2,3, \ldots$ Let $B$ be (unique) minimal for $B \triangleleft G$, $G / H \in \underline{\underline{N}}^{h(G)-2}$, and $H / K$ a chief factor of $G$. Clearly $F \subset K<H$ and $H / K$ is a $p^{\prime}$-group. Moreover there exists for $h(G)>2$, a maximal subgroup $M$ of $G$ complementing $H / K$, by [1]. Let $R=C_{G}(H / K)$, $S=R \cap M$. Then $C_{G}(H / K)=C_{G}(R / S), G / C_{G}(R / S) \notin N^{h(G)-3}$ and so $h(G / S)=h(G)-1$. For $h(G)=2$, we may take any maximal normal subgroup of $G$ for $S$.

Proof of the Theorem. Let $N_{1}, N_{2}$ be two different minimal normal subgroups of $G$. Then $h(G)=\max \left(h\left(G / N_{1}\right), h\left(G / N_{2}\right)\right) \leqq \max \left(f\left(\nu\left(G / N_{1}\right)\right)\right.$, $\left.f\left(\nu\left(G / N_{2}\right)\right)\right)$, by induction. If $N$ is a minimal normal subgroup of $G$, and $N \subset \Phi(G)$, then $h(G)=h(G / \Phi(G))$, hence $h(G)=h(G / N) \leqq f(\nu(G / N))$, by induction. Thus, if we can find an increasing function $f(v)$ which bounds $h(G)$ for all groups $G$ having their Fitting subgroups as unique minimal normal subgroups, $f(v)$ is then a general bound for $h(G)$. Hence let us assume that $G$ has its Fitting subgroup $F$ as its unique minimal normal subgroup, and suppose $F$ is a p-group. There is exactly one conjugacy class of maximal nilpotent subgroups of $G$ containing $F$, namely the Sylow $p$-subgroups of $G$. Hence by Lema 3 , every abelian $q$-subgroup, $q \neq p$, can be generated by at most $v(G)-1$ elements. Lemma 4 implies that the $p^{\prime}$-chief factors of $G$ are of rank at most 
$\frac{\nu(G)(\nu(G)-1)}{2}$. We choose $S \triangleleft G$ accordingly to Lemma 6 , and Lemma $I$ implies $\nu(G / S) \leqq \nu(G)$. Hence, all $p$-chief factors of $G / S$ are of rank at most $\frac{\nu(G)(\nu(G)-1)}{2}$. Therefore, by [6], VI. Hauptsatz $6.6 \mathrm{c}$, the p-length of $G / S$ is at most $\frac{\nu(G)(\nu(G)-1)}{2}$. Assume $\nu(G)>2$. Let $\left\{p_{1}, p_{2}, \ldots, p_{r(v)}\right\}$ be the set of all odd primes less than or equal to $\frac{v(G)(\nu(G)-1)}{2}$, take the upper $p_{1}$-series of $G / S$ refine each factor by a $p_{2}$-series, etc. One obtains a normal series of $G / S$ of length at most $2(s(v)+1)^{r(v)}-1$ where $s(\nu)=\frac{\nu(G)(\nu(G)-1)}{2}$, consisting of $p_{i}$-factors, $i=1,2, \ldots, r(\nu)$, and $\left\{p_{1}, p_{2}, \ldots, p_{r(v)}\right\}^{\prime}$-factors. By Lema 5 , these $\left\{p_{1}, p_{2}, \ldots, p_{r(v)}\right\}^{\prime}-$ factors are all of Fitting length at most 2 , and there are at most $(s(v)+1)^{r(v)}$ of them. Hence, $h(G / S) \leqq 3(s(v)+1)^{r(v)}-1$ and $h(G) \leqq 3(s(v)+1)^{r(v)}$. Since $s(v)$ and $r(v)$ are increasing functions, we may take $f(\nu)=3(s(\nu)+1)^{r(\nu)} . v(G)=1$ implies $h(G)=1$, for $v(G)=2,\left\{p_{1}, \ldots, p_{r(v)}\right\}=\varnothing$, and again Lemma 5 implies that $h(G / S) \leqq 2$ whence $h(G) \leqq 3 \cdot$ Q.E.D.

COROLLARY. If $q|| G \mid$ implies $q>\frac{\nu(G)(\nu(G)-1)}{2}$, then $h(G) \leqq 3$.

Proof. This is an immediate consequence of Lemma 5 , provided $|G|$ is odd. Now assume $\nu(G)=2$, and 2||$G \mid$. We may assume that $G$ has its Fitting subgroup $F$ as its unique minimal normal subgroup. First, let $E$ be a 2-group. Lemma 3 implies $G_{2}$, is cyclic, and a Hall-Higman type argument [4] shows that the' $2^{\prime}$-length of $G$ is at most 1 whence $h(G) \leqq 3$. Now let $F$ be a $2^{\prime}$-group. Lemma 3 implies that $G_{2}$ is cyclic or a generalized quaternion group. In either case $G / F$ possesses a characteristic subgroup of order 2 which is clearly central in $G / F$. Assume $G / F$ is not nilpotent and $F$ is a p-group, $p \in 2^{\prime}$. Then $G / F$ contains an element $x F$ of order $2 p$. Let $x=y z, o(y)=p^{\alpha}$, $o(z) \in p^{\prime}$. Then $y \in C_{F}\left(G_{p^{\prime}}\right)$ for some $p$-complement $G_{p^{\prime}}$ of $G$, and $x F=z F$ is a $p^{\prime}$-element, contradiction. Hence, in this case, $h(G) \leqq 2$.

3. For $\nu(G)=2, h(G)=3$ is really attained for some groups $G$. The symmetric group $S_{4}$ on 4 letters provides such an example. Moreover, let $H=C_{q} C_{p}$ be a (non-direct) semidirect product of a group $C_{q}$ of order 
$q$ by a group $C_{P}$ of order $p$ and let $G=\bar{C}_{p}$ wr $H$ be the wreath product of a group $\bar{C}_{p}$ of order $p$ by $H$. Then $\nu(G)=2$, and $h(G)=3$ as one can easily check.

For groups minimal for $v(G)=2, h(G)=3$ we get the following result:

PROPOSITION 7. Let $G$ be a finite group which is minimal with respect to the property that $\nu(G)=2$ implies $h(G)=3$. Then $|G|=p^{\alpha} q^{\beta}$, $p, q$ being distinct primes, and $G$ contains no element of order $p q$. In particular, if $F$ is the Fitting subgroup of $G$, then $|F|=p^{\gamma}$, for some $\gamma>0$, and $\beta=1, \gamma=\alpha-1$.

REMARK. Since $\nu(G)=2, G=V_{1} V_{2}$ where $V_{1} V_{2}$ are maximal nilpotent subgroups of $G$. In this case, the solubility of $G$ follows from a theorem of Wielandt and Kegel [11].

First we prove

LEMMA 8. Let $G$ be a finite group minimal with the properties that $v(G)=2$ implies $h(G)=3$. Then the maximal nilpotent subgroups of $G$ are Hall subgroups of $G$.

Proof. Let $V_{1}$ and $V_{2}$ be representatives of the two conjugacy classes of maximal nilpotent subgroups of $G$. By hypothesis, we may assume that $G$ has its Fitting subgroup $F$ as its unique minimal normal subgroup. Without loss of generality, we may assume $V_{1} \supset F$. Let $|F|=p^{\gamma}$, then $V_{1}=G_{p}$, a sylow $p$-subgroup of $G$, and $V_{2}=G_{p^{\prime}} \times C_{G_{p}}\left(G_{p^{\prime}}\right)$ where $G_{p^{\prime}}$ is a p-complement of $G$. We have to show $C_{G}\left(G_{p^{\prime}}\right)=1$. Let $F_{2} / F$ be the Fitting subgroup of $G / F$, then $F_{2} / F \cong G_{p^{\prime}}$, moreover $C_{G_{p}}\left(G_{p^{\prime}}\right)=C_{F^{\prime}}\left(G_{p^{\prime}}\right)$. Also $C_{F}\left(G_{p^{\prime}}\right) \subset Z\left(F_{2}\right)$ since $F$ is abelian. We claim $Z\left(F_{2}\right)=C_{F^{\prime}}\left(G_{p^{\prime}}\right)$. For $Z\left(F_{2}\right)=$ $\left(Z\left(F_{2}\right)\right)_{p^{\prime}} \times C_{F}\left(G_{p^{\prime}}\right)$ where $\left(Z\left(F_{2}\right)\right)_{p^{\prime}}$ is the $p^{\prime}$-complement of $Z\left(F_{2}\right)$. As $F$ is self-centralizing it follows $\left(Z\left(F_{2}\right)\right)_{p^{\prime}}=1$. Thus $C_{F^{\prime}}\left(G_{p^{\prime}}\right)=2\left(F_{2}\right)$ char $F_{2} \triangleleft G$ implying $C_{F^{\prime}}\left(G_{p^{\prime}}\right)=1$ as $F$ is a minimal normal subgroup of $G$. 
Proof of Proposition 7. By Lemma 8, $V_{1}=G_{p}, V_{2}=G_{p}$, . Suppose $q|| V_{2} \mid$. Therefore $G$ contains no element of order $p q$, otherwise $\nu(G)>2$, and so $G / F$ contains no element of order $p q, q \in p^{\prime}$. Certainly $V_{2} F=F_{2}$ as $h(G / F)=2$. Suppose $V_{2}$ is not a Sylow $q$-subgroup for some $q \in p^{\prime}$. Then, for some prime $r$, let $R$ be a Sylow $r$-subgroup of $G, r \neq q$. Then $F R$ char $F_{2}$ and so $F R \triangleleft G$. Consider $G_{1}=(F R) V_{1}=R V_{1} \cdot R V_{1}$ contains no element of order $p r$, therefore $V\left(G_{1}\right)=2, G_{1}<G$. Minimality of $G$ implies $h\left(G_{1}\right)=2$. But then $F R=G_{1}$ since $F$ is self-centralizing and so $V_{1}=F$, hence $h(G)=2$, contradiction. Thus $V_{2}=G_{q}$, a Sylow q-subgroup of $G$, and $|G|=\left|V_{1} V_{2}\right|=p^{\alpha} q^{\beta} . \quad V_{2}$ acts in a fixed-point-free manner on $F$, hence $V_{2}$ is cyclic $(q=2$ can be excluded, for then $h(G)=2$, by the proof of the corollary of the theorem). Let $S / F$ be the cyclic normal subgroup of index $q$ in $F_{2} / F$. Then $S \triangleleft G$. Using the same argument as above, we may conclude $V_{1}=F$ provided $\beta>1$. Therefore $\beta=1$. Let $M$ be a maximal subgroup of $G$ containing $F_{2}$. Then $M \triangleleft G$, $V_{1} \cap M$ is a sylow p-subgroup of $M$, and $M$ contains no element of order $p q$. Therefore $\nu(M)=2$. Minimality of $G$ implies $h(M)=2$ and so $M=F_{2}$. Therefore $\gamma=\alpha-1$.

4. We are going to give an exact bound for $h(G)$ in the case of $v(G)=3, \quad|G|$ odd.

LEMMA 9 (Thompson). Suppose $p$ is an odd prime, $G$ is a p-soluble group and $G$ has no elementary-abelian subgroup of order $p^{3}$. Then each $p$-chief factor of $G$ is of order $p$ or $p^{2}$.

Proof. See [10].

COROLLARY. If $|G|$ is odd, $\nu(G)=3$, then $h(G) \leqq 3$.

Proof. By Lemmas 3, 5, and 6 .

\section{References}

[1] Roger Carter and Trevor Hawkes, "The $\mathcal{F}$ normalizers of a finite soluble group", J. Algebra 5 (1967), 175-202.

[2] Daniel Gorenstein, Finite Groups (Harper and Row, New York, Evanston, London, 1968). 
[3] Fletcher Gross, "On finite groups of exponent $p^{m} q^{n}$, J. Algebra 7 (1967), 238-253.

[4] P. Hall and Graham Higman, "On the p-length of p-soluble groups and reduction theorems for Burnside's problem", Proc. London Math. Soc. (3) 6 (1956), 1-42.

[5] Frederik Hoffman, "Nilpotent height of finite groups admitting fixedpoint-free automorphisms", Math. Zeitschr. 85 (1964), 260-267.

[6] Bertram Huppert, EndZiche Gmppen I. (Berlin, Heidelberg, New York, 1967).

[7] Bertram Huppert, "Zur Gaschützschen Theorie der Formationen", Math. Ann. 164 (1966), 133-141.

[8] John S. Rose, "Nilpotent subgroups of finite soluble groups", Math. Zeitschr. 106 (1968), 97-112.

[9] John G. Thompson, "Automorphisms of solvable groups", J. AZgebra 1 $(1964), 259-267$.

[10] John G. Thompson, "Nonsolvable finite groups all of whose local subgroups are solvable", Buzl. Amer. Math. Soc. 74 (1968), 383-437.

[11] Otto H. Kegel, "Produkte nilpotenter Gruppen", Arch. Math. 12 (1961), 90-93.

Department of Mathematics, IAS, The Australian National University, Canberra, A.C.T. 